\title{
Microbiological Hydroxylation of Steroids by Absidia regnieri
}

Sir :

In microbial hydroxylations of steroids, the introduction of the 14-hydroxy group is a common reaction caused by molds, especially by the species of Mucor ${ }^{1}$.

We have also found that a strain of Absidia regnieri carries out the $14 \alpha$-hydroxylation of steroids. When progesterone was used as the steroid substrate in the fermentation of Absidia regnieri, $14 \alpha$-hydroxyprogesterone was obtained as the monohydroxyderivative of the steroid substrate.

But besides this monohydroxylation, this fungus was also capable of introducing, at the same time, two or three hydroxyl groups into progesterone.

The introduction of three hydroxyl groups at the same time into the steroid nucleus rarely occurs in microbial hydroxylation.

Hereupon, in the present communication we wish to report on the preparation of $14 \alpha$-hydroxyprogesterone, $6 \beta, 14 \alpha-; 7 \alpha, 14 \alpha$-dihydroxyprogesterone and $7 \alpha, 14 \alpha, 15 \beta$-trihydroxyprogesterone by Absidia regnieri.

When Reichstein's compound S (17 $\alpha, 21$-dihydroxy-4-pregnene-3,20-dione) was used instead of progesterone in fermentation of this fungus, it was converted predominantly to epi-hydrocortisone $\quad(11 \alpha, 17 \alpha, 21$-trihydroxy-4-pregnene3,20-dione), making only minor amounts of the 14 $\alpha$-hydroxyderivative.

This result is particularly interesting in respect of stereochemical specificity of substrates on the microbial hydroxylation of steroid.

In our experiment reported here, Absidia regnieri was grown on a reciprocating shaker for 48 hours at $26^{\circ}$ in 101 of a medium of the

\footnotetext{
1) P. D. Meister, S. H. Eppstein et al., J. Am. Cbem. Soc, $\mathbf{8 0}$, 3383 (1958).
}

following composition: $5 \%$ glucose, $2 \%$ pepton and $0.5 \%$ corn steep liquor.

Then, 5 grams of progesterone was added in a methanol solution and fermentation was allowed to proceed for additional 48 to 72 hours.

The culture was filtered and the oxydized steroids were recovered from the filtrate by ethyl acetate extraction followed by washing with $2 \%$ sodium bicarbonate solution and water. The ethyl acetate solution was evaporated to dryness in vacuo and redissolved in chloroform and the insoluble matter was removed by filtration. The product obtained by recrystallization of this insoluble matter from acetone was $6 \beta, 14 \alpha-$ dihydroxyprogesterone (I), m.p. 242-246 ${ }^{\circ} ;[\alpha]_{\mathrm{D}}+$ $132^{\circ}$ (pyridine); $\lambda_{\max }^{\text {ale. }}, 236 \mathrm{~m} \mu(\varepsilon=13,800)$. The monoacetate of (I), m.p. 175.5-177.5 , $[\alpha]_{\mathrm{D}}$ $+101.8^{\circ}$ (pyridine).

Reduction of (I) by mild treatment with zinc and acetic acid afforded a known-14 $\alpha$-hydroxyprogesterone.

The chloroform solution of the residue was chromatographed on Florisil and developed by chloroform-acetone solvent mixtures.

The major product eluted in the first fraction was $14 \alpha$-hydroxy progesterone (II), m.p. 192-194\%; $[\alpha]_{\mathrm{D}}+197^{\circ}$ (chloroform); $\lambda_{\max }^{\text {alc. }} 240 \mathrm{~m} \mu(\varepsilon=17,050)$.

From the second fraction was yielded $7 \alpha, 14 \alpha-$ dihydroxyprogesterone (III), m.p. 212-216 $;[\alpha]_{\mathrm{D}}$ $+141^{\circ}$ (chloroform); $\lambda_{\text {max. }}^{\text {ale. }} 242 \mathrm{~m} \mu(\varepsilon=16,200)$. The monoacetate of (III), m.p. 191.5-192.5\%; $[\alpha]_{D}+161.0^{\circ}$ (chloroform). The crystallization of the last group of fractions afforded trihydroxyprogesterone (IV), m.p. 267-269 ${ }^{\circ}$ (dec.); $[\alpha]_{\mathrm{D}}+$ $75.1^{\circ}$ (pyridine); $\lambda_{\max }^{\text {ale. }} 239.5 \mathrm{~m} \mu(\varepsilon=16.020)$. The infrared spectrum of (IV) was idential to that of $7 \alpha, 14 \alpha, 15 \beta$-trihydroxyprogesterone ${ }^{2 y}$ obtained 2) K. Tsuda, E. Oki et al., Monthly Meeting of Pharm. Soc. Japan (Tokyo). Dec. 6, 1958. 
by a strain of Syncephalastram sp.

Some other unidentified dihydroxyprogesterone was also obtained in a small amount.

When Reichstein's compound S was used as asteroid substrate in similar techniques, most of the oxygenated product obtained was epi-hydrocortisone (V), m.p. $206-209^{\circ} ;[\alpha]_{\mathrm{n}}+120^{\circ}$ (methanol), $\lambda_{\text {mix. }}^{\text {llc. }} 242 \mathrm{~m} \mu \quad(\varepsilon=14,500)$ and a minor amount of $14 \alpha, 17 \alpha, 21$-trihydroxy-4-pregnene3,20-dione (VI) was isolated, m.p. 213-218 $[\alpha]_{\mathrm{D}}+155^{\circ}$ (methanol); $2_{\max }^{\text {alc. }} .24 \mathrm{l} \mathrm{m} \mu(\varepsilon=11,000)$. The 21-mono-acetate of (VI) was, m.p. 222-227 $[\alpha]_{D}+177^{\circ}$ (chloroform). From these results, this fungus has considerable stereochemical specificity of steroid substrate in hydroxylation, that is, it appears that $14 \alpha$-hydroxylation by this fungus is affected profoundly by the presence of $17 \alpha$-hydroxyl or the 21-hydroxyl function.

The authors wish to express their thanks to Professor K. Tsuda and Professor T. Asai, of the Institute of Applied Microbiology, University of Tokyo, and Mr. M. Matsui, Director of this Laboratory for their helpful advice and constant guidance throughout this work.

Makoto Shirasaka Ryozo Hayashi Masako Tsuruta

Takamine Laboratory, Sankyo, Co., Ltd.

Received March 5, 1959

[Bull. Agr. Chem. Soc. Japan, Vol. 23, No. 3, p. 245 246, 1959]

\section{Microbiological Hydroxylation of Progesterone and $17 \alpha$-Hydroxyprogesterone by Sclerotinia libertiana; $2 \beta-H$ Hdroxylation.}

Sir :

Among the numerous hydroxylation of steroids by microorganisms, $2 \beta$-hydroxylation has only recently been discovered".

In the previous paper ${ }^{2}$ we have reported on a microbial hydroxylation of Reichstein's compound $S$ (17 $\alpha$-hydroxydesoxycorticosterone) to $2 \beta, 17 \alpha, 21$-trihydroxy-4-pregnene-3,20-dione utilizing Sclerotinia libertiana.

In the present communication we wish to report on the microbial hydroxylation of progesterone and $17 \alpha$-hydroxyprogesterone by this fungus in the $2 \beta$-position.

When progesterone was used as the steroid substrate, the fermentation of this fungus yielded $2 \beta, 15 \beta$-dihydroxyprogesterone and several unidentified dihydroxyprogesterones.

In case $17 \alpha$-hydroxyprogesterone was used, $2 \beta, 17 \alpha$-dihydroxy-, $15 \beta, 17 \alpha$-dihydroxy-and $11 \alpha$, $17 \alpha$-dihydroxyprogesterone were produced.

1) H. L. Herzog, J. Am. Chem. Soc., 79, 3921 (1957).

G. Greenspan et al., ibid.., 79, 3922 (1957).

2) M. Shirasaka et al., Bull. Agr. Cbem. Sor. Japan, 22, 273 (1958).
Among these isolated compounds, $2 \beta, 15 \beta$ dihydroxy- and $2 \beta, 17 \alpha$-dihydroxyprogesterone have been recognized to be hitherto- undescribed new compounds.

In the experiment reported here, Sclerotinia libertiana was grown aerobically on a reciprocating shaker at $26^{\circ}$ for 48 hours in 101 of a medium of potatodecoct containing $3 \%$ of glucose.

Five grams of the requisite steroid was then added in methanol solution and the fermentation was allowed to proceed for a further 72 hours. The oxygenated products were extracted with ethyl acetate from the whole broth and the extract was first washed with $2 \%$ sodium bicarbonate solution and then with water. The ethyl acetate solution was then taken to dryness in vacuo and the residue was dissolved in chloroform. This chloroform solution was chromatographed on Florisil or alumina. Chloroformacetate solvent mixtures were used as the developer.

When progesterone was incubated with Sclero- 